\title{
Cardiac resynchronization therapy pacemaker: critical appraisal of the adaptive CRT-P device
}

This article was published in the following Dove Press journal:

Medical Devices: Evidence and Research

18 January 2016

Number of times this article has been viewed

\section{Georges E Daoud' Mahmoud Houmsse ${ }^{2}$ \\ 'Department of the Biomedical Research, ${ }^{2}$ Division of Cardiovascular Medicine, The Ohio State University Wexner Medical Center, Columbus, $\mathrm{OH}$, USA}

Correspondence: Mahmoud Houmsse Division of Cardiovascular Medicine, Davis Heart and Lung Research Institute, The Ohio State University Wexner Medical Center, Suite 200, 472 W

I2th Avenue, Columbus, OH 43210, USA

$\mathrm{Tel}+\mathrm{I} 6 \mid 42934967$

Fax + I 6I4 2935614

Email mahmoud.houmsse@osumc.edu
Abstract: Cardiac resynchronization therapy (CRT) is an effective and well-established therapy for patients suffering with heart failure, left ventricular (LV) systolic dysfunction (ejection fraction $\leq 35 \%$ ), and electrical dyssynchrony, demonstrated by a surface QRS duration of $\geq 120 \mathrm{~ms}$. Patients undergoing treatment with CRT have shown significant improvement in functional class, quality of life, LV ejection fraction, exercise capacity, hemodynamics, and reverse remodeling of LV, and ultimately, morbidity and mortality. However, $30 \%-40 \%$ of patients who receive a CRT device may not show improvement, and they are termed as nonresponders. The nonresponders have a poor prognosis; several methods have been developed to try to enhance response to CRT. Echocardiography-guided optimization of CRT has not resulted in significant clinical benefit, since it is done at rest with the patient in supine position. An ideal optimization strategy would provide continuous monitoring and adjustment of device pacing to provide maximal cardiac resynchronization, under a multitude of physiologic states. Intrinsic activation of the right ventricle (RV) with paced activation of the RV, even in the setting of biventricular (BiV) pacing, may result in an adverse effect on cardiac performance. With this physiology, the use of LV-only pacing may be preferred and may enhance CRT. Adaptive CRT is a novel device-based algorithm that was designed to achieve patient-specific adjustment in CRT so as to provide appropriate $\mathrm{BiV}$ pacing or LV-only pacing. This article will review the goals of CRT optimization, and implementation and outcomes associated with adaptive CRT.

Keywords: heart failure, cardiac resynchronization, therapy, adaptive, left and biventricular pacing

\section{Introduction}

Cardiac resynchronization therapy (CRT) is an effective and well-established therapy for patients suffering with heart failure (HF), left ventricular (LV) systolic dysfunction (ejection fraction $\leq 35 \%$ ) and electrical dyssynchrony, demonstrated by a surface QRS duration of $\geq 120 \mathrm{~ms}$. Patients undergoing treatment with CRT have shown significant improvement in functional class, quality of life, LV ejection fraction (EF), exercise capacity, hemodynamics, and reverse remodeling of LV, and ultimately, morbidity and mortality. However, $30 \%-40 \%$ of patients who receive a CRT device may not show improvement, and they are termed as nonresponders. The reasons for being a nonresponder are numerous and include, but are not limited to, scar burden and distribution, LV stimulation site, and limited electrical dyssynchrony. ${ }^{1,2}$

Considering the risks and costs associated with implantation of a CRT device, and that nonresponders have a poor prognosis, several methods have been developed to try to enhance response to CRT. Echocardiography-guided optimization of 
atrioventricular (AV) delay and interventricular (VV) delay between the LV and right ventricle (RV) was enthusiastically used to guide device programming. Unfortunately, though, echocardiography optimization (ECHO) has not resulted in significant clinical benefit because it is a time-consuming, resource-draining process and the parameters measured were often at rest with the patient in supine position. ${ }^{3}$ An ideal optimization strategy would provide continuous monitoring and adjustment of device pacing to provide maximal cardiac resynchronization, under a multitude of physiologic states.

Another important observation in device trials is that in the presence of intact $\mathrm{AV}$ conduction, $\mathrm{LV}$ dysfunction, and a narrow QRS, RV pacing results in deleterious effects due to pacing-induced dyssynchrony with subsequent increase in HF events and deterioration in left ventricular ejection fraction (LVEF). ${ }^{4}$ To date, the primary therapy of CRT has been simultaneous BiV pacing (ie, VV delay of 0 ); however, in the setting of intact AV conduction, this programming of CRT results in delivery of RV pacing, and thus substitutes intrinsic RV activation with RV-paced activation. Replacing intrinsic activation of the RV with paced activation of the RV, even in the setting of BiV pacing, may result in an adverse effect on cardiac performance. With this physiology, the use of LV-only pacing may be preferred and may enhance CRT.

Adaptive CRT (aCRT) is a novel device-based algorithm that was designed to achieve patient-specific adjustment in CRT so as to provide appropriate BiV pacing or LV-only pacing. ${ }^{5-8}$ This article will review the goals of CRT optimization, and implementation and outcomes associated with aCRT.

\section{Optimization}

Recognizing that up to $40 \%$ of patients fail to respond to CRT, there are numerous studies that have assessed methods to optimize response to $\mathrm{BiV}$ pacing, including lead position and echocardiography-guided programming of $\mathrm{AV}$ and $\mathrm{VV}$ intervals.

Rossillo et al evaluated 233 consecutive patients with New York Heart Association (NYHA) class III-IV HF and LVEF $<35 \%$ who had successful placement of a transvenous LV lead. ${ }^{9}$ Outcomes (functional capacity and LVEF) were significantly improved in patients in whom the lead was placed in the lateral/posterolateral branch in comparison to the anterior and anterolateral branch.

Optimal AV delay has been an integral part of CRT therapy. Suboptimal AV delay programming can result in a $10 \%-15 \%$ decline in cardiac output; yet, the methods of optimization are varied: no optimization (CONTAK CD trial);
Doppler echocardiography of transmitral flow (CARE-HF and MIRACLE trials); and, calculation of AV delay based on intrinsic AV delay and QRS duration (COMPANION trial). The SMART-AV trial was designed to compare a device-based algorithm, the SmartDelay, to echocardiography-guided AV delay optimization and to fixed nominal AV delay in a randomized pattern enrolling 1,014 patients. ${ }^{10}$ The primary end point in this study was LV end-systolic volume, and secondary end points included NYHA class, quality-of-life score, 6-minute walk distance, LV end-diastolic volume, and LVEF. The study concluded that neither SmartDelay nor echocardiography guidance was superior to a fixed AV delay of $120 \mathrm{~ms}$. One likely explanation for why these sophisticated methods of AV optimization do not achieve improved results compared to nominal device programming is due to the dynamic nature of AV node physiology, at rest and with exertion. Considering the time and expense related to echocardiography-guided optimization, this technique is rarely utilized. Although maximizing $\mathrm{AV}$ timing offers important physiologic benefit and other studies have suggested that systematic AV interval optimization improves clinical outcomes, to date, there is no algorithm that reproducibly and reliably enhances AV timing with subsequent persistent hemodynamic benefit.

A third method to achieve optimal outcome from CRT is to alter VV timing. VV timing refers to the timing of delivery of electrical stimulus to the right and left ventricular leads, so as to alter interventricular activation. Conventional CRT is to deliver the stimulus simultaneously, BiV-0. In a prospective trial, enrolling 274 patients with NYHA class III-IV HF and who underwent CRT implantation, VV timing was randomized to simultaneous VV pacing (BiV-0) or to optimized VV timing based on echocardiography-guided assessment of LV septal to posterior wall motion delay during CRT. ${ }^{11}$ End points included a clinical composite end point of mortality: HF hospitalization, NYHA functional class, and patient global assessment, as well as 6-minute hall walk distance, quality of life, peak oxygen uptake, and event-free survival. The study found only modest but inconsistent benefit with optimized sequential VV stimulation, and this finding is similar to other studies investigating VV timing.

Methods to guide CRT device programming to enhance response to $\mathrm{BiV}$ pacing may have clinical relevance for those patients who do not respond to CRT; however, even among a population of nonresponders, the current techniques for optimization are not reliable or effective to manage the dynamic nature of cardiac conduction. Cardiac electrical events are altered by autonomic tone, drug therapy, ventricular volume/ loading, and varied other factors such as exercise or upright 
posture. Optimization with one-time CRT device programming will not offer a lasting effect and, more importantly, will not respond to changes in cardiac conduction under a broad spectrum of conditions. Furthermore, although CRT is typically achieved with simultaneous $\mathrm{BiV}$ pacing, some studies have confirmed the value of LV-only pacing, which has been demonstrated to be at least as efficacious as $\mathrm{BiV}$ pacing and may be more effective. Other reports have evaluated the impact of LV pacing that is synchronized to RV activation in the setting of intact $\mathrm{AV}$ and right bundle branch conduction. ${ }^{12}$ LV pacing synchronized to normal RV activation produces electrical fusion of pacing activation of the LV with native $\mathrm{RV}$ activation and has been demonstrated to result in superior LV/RV stimulation and function compared to simultaneous BiV pacing.

A device-based algorithm that continuously alters device stimulation, LV only synchronized with native RV activation or BiV pacing, relative to measured intracardiac events is more likely to provide a dynamic physiologic response so as to maximize CRT, for responders as well as for nonresponders.

\section{Adaptive CRT algorithm}

Adaptive CRT (aCRT) is a novel pacing algorithm to provide continuous, dynamic, and ambulatory optimal CRT by measuring intracardiac conduction parameters, and then responding with either LV-only pacing, synchronized to $\mathrm{RV}$ activation, or simultaneous BiV pacing in the setting of abnormal AV/right bundle conduction. In this manner, the algorithm was developed to minimize RV pacing and the associated pacing-induced dyssynchrony, and in turn, to minimize nonresponders and to improve efficacy of CRT.

Adaptive CRT works by constantly measuring intracardiac timing events and altering pacing in response to three elements: intrinsic conduction, determination of the best pacing (LV-only or $\mathrm{BiV}$ ), and optimization of $\mathrm{AV}$ and $\mathrm{VV}$ timing, in that sequence.

\section{Intrinsic conduction (AV interval, P-wave, and QRS interval)}

$\mathrm{AV}$ conduction interval is measured every minute by extending the AV delay to $300 \mathrm{~ms}$ for one beat. The timing is from either atrial sensed or atrial paced event to RV sensing (As$\mathrm{RVs}$ or Ap-RVs). The $\mathrm{P}$-wave conduction timing is measured from atrial sensing to the end of $\mathrm{P}$-wave on the far-field atrial electrogram (A-Pend). The QRS duration is measured from RVs to the end of QRS on the far-field electrogram (RVsQRSend). From these measurements, the P-wave interval and QRS duration are measured every 16 hours.
Determination of the best pacing method First, capture of the LV lead is confirmed. Second is the determination of adaptive $\mathrm{LV}$ pacing or adaptive $\mathrm{BiV}$ pacing. If the sensed AV interval, As-RVs, is $\leq 200 \mathrm{~ms}$, or if the paced AV delay (Ap-RVs) is $\leq 250 \mathrm{~ms}$, and heart rate is $<100 \mathrm{bpm}$, then pacing is with adaptive $\mathrm{LV}$ pacing.

However, if the As-RVs is $>200 \mathrm{~ms}$, or the Ap-RVs $>250 \mathrm{~ms}$, and the heart rate is $>100 \mathrm{bpm}$, then adaptive Biv pacing is delivered.

\section{Optimization of $A V$ and $V V$ timing}

If adaptive LV pacing is selected, then the LV will pace at either $70 \%$ of the measured AV interval (As-RVs/Ap-RVs interval) or $40 \mathrm{~ms}$ before intrinsic A-RVs interval, whichever value is smaller. If adaptive $\mathrm{BiV}$ pacing is delivered, then $\mathrm{BiV}$ pacing will be $30 \mathrm{~ms}$ after the end of $\mathrm{P}$-wave or $50 \mathrm{~ms}$ before intrinsic A-RVs interval, whichever is smaller.

The interventricular pacing delay (VV timing) during BiV pacing is calculated based on A-RVs as well as RVs-QRS end intervals. The shortest VV interval will be selected to allow LV preactivation. However, if the QRS is $>160 \mathrm{~ms}$, then BiV-0 (simultaneous delivery of current to the RV and LV leads) pacing will be selected.

With these three steps, the goal of aCRT is to continuously measure the AV interval utilizing RV sensing (Figure 1). ${ }^{8}$ If right atrium to RV conduction is intact and normal, as represented by AV node + right bundle conduction, the algorithm provides $\mathrm{LV}$-only pacing. In this manner, $\mathrm{LV}$ pacing is

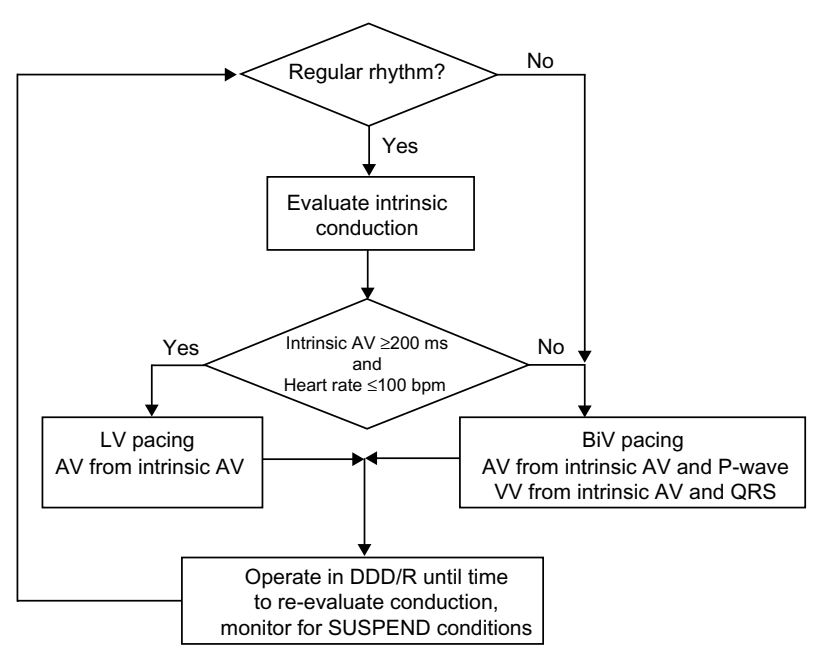

Figure I Summary of aCRT algorithm.

Notes: Reprinted from American Heart Journal, I63(5), Krum H, Lemke B, Birnie D, et al. A novel algorithm for individualized cardiac resynchronization therapy: rationale and design of the adaptive CRT trial, 747-752. Copyright (2012), with permission from Elsevier. ${ }^{8}$

Abbreviations: aCRT, adaptive cardiac resynchronization therapy; AV, atrioventricular; LV, left ventricular; BiV, bi-ventricular; VV, interventricular; DDD/R, dual chamber pacemaker with and without rate response. 
synchronized to RV native activation to produce fusion with native right bundle activation. Since electrical conduction, particularly via the AV node, is dynamic, the AV delay is adjusted to produce optimal fusion with intrinsic activation. In this manner, the RV will be activated via intrinsic conduction, which averts the adverse physiology and dyssynchrony related to RV pacing. In the circumstances when $\mathrm{AV}$ conduction is poor, then $\mathrm{BiV}-0$ pacing is provided with electrical stimulation based on optimizing AV delay, utilizing timing from the end of the P-wave. This aCRT algorithm is suspended during any sustained atrial or ventricular tachycardia, and is not applicable in the setting of complete heart block or AV junction ablation. Unpublished data about the accuracy of the automatic and manual measurement of the previously mentioned waves and intervals showed a mean error of $7 \mathrm{~ms}$ only in P-wave measurement (Medtronic Inc., personal communication, October, 2015).

\section{Clinical trials with aCRT}

The primary study investigating aCRT was by Martin et al, published in 2012. ${ }^{13}$ This was a prospective, multicenter, randomized, double-blind, "noninferiority" trial comparing outcomes of aCRT algorithm programmed ON versus simultaneous BiV pacing optimized by echocardiography. The study randomized 478 patients with HF who met criteria for de novo CRT system (NYHA Class III or IV symptoms, $\mathrm{LVEF} \leq 35 \%$, and $\mathrm{QRS} \geq 120 \mathrm{~ms}$ ). All patients first underwent an echocardiography-based AV and VV timing optimization. VV timing was guided by aortic velocity time integral (AoVTI), to measure stroke volume, and AV optimization by the iterative method. After echo-guided optimization, the aCRT software was downloaded into all devices. Patients were then randomized in a 2:1 manner to chronic pacing using aCRT versus ECHO.

Study follow-up was at 1, 3, and 6 months and then every 6 months thereafter. There were three primary end points at 6 months: 1) the clinical composite score (CCS) - an aggregate measure of death, hospitalization, and change in NYHA class, and patients were classified as improved, unchanged, or worsened; 2) the concordance correlation coefficient (CCC) between the AoVTI values measured for each patient under echo-optimized and aCRT exceeded 0.82 both at randomization and at 6-month post-randomization; and 3 ) safety of the ambulatory aCRT algorithm. Safety was defined as no more than $60 \mathrm{~ms}$ variance of the AV or VV delays throughout a 28 -day period. If all primary end points were met, secondary end points were analyzed including: reduction of RV pacing in the aCRT arm, death, HF hospitalization, ventricular arrhythmias, changes in LV end-systolic

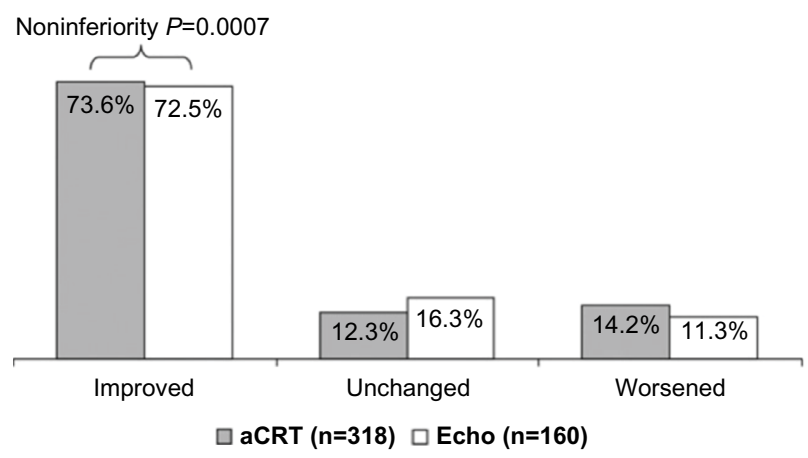

Figure 2 Summary of aCRT study.

Notes: Reprinted from Heart Rhythm, 9(II), Martin DO, Lemke B, Birnie D, et al. Investigation of a novel algorithm for synchronized left ventricular pacing and ambulatory optimization of cardiac resynchronization therapy: results of the adaptive CRT trial, 1807-18I4. Copyright (2012), with permission from Elsevier. ${ }^{13}$ Abbreviation: aCRT, adaptive cardiac resynchronization therapy.

volume index, LVEF, NYHA classification, 6-minute hall walk distance, and quality of life.

The aCRT study met all the three noninferiority primary end points. The percentage of patients who improved in the CCS at 6 months was $73.6 \%$ for aCRT versus $72.5 \%$ for ECHO (Figure 2; noninferiority $P<0.0007$ ); the CCC at randomization and at 6-month follow-up was high, 0.93 and 0.90 , respectively (Figure 3); and aCRT did not result in inappropriate device settings. During a mean follow-up of 9.7 months, there were no differences in mortality or HF events and no difference in time to first occurrence of HF or of ventricular arrhythmias between the study groups. Other secondary end points are summarized in Table 1. In the ECHO group, nearly all ventricular pacing was $\mathrm{BiV}$, whereas in the aCRT group, median of the occurrence of $\mathrm{BiV}$ pacing was $51 \%$ and the remainder was LV-only pacing, with a $44 \%$ reduction in RV pacing. The study concluded that aCRT was safe and noninferior to echocardiography-guided optimization.

Two post hoc analysis procedures were considered for the aCRT trial. The first study compared the treatment arm of aCRT ( $\mathrm{n}=266)$ to a pooled historical control $(\mathrm{HC})$ group
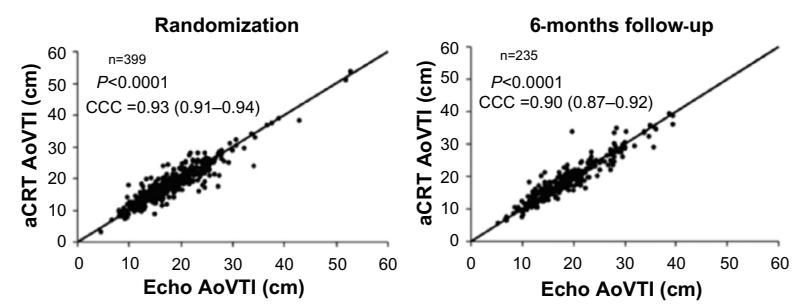

Figure 3 Summary of aortic velocity time integral (AoVTI) at randomization and at 6-months follow-up comparing aCRT to echo-guided optimization.

Notes: Reprinted from Heart Rhythm, 9(II), Martin DO, Lemke B, Birnie D, et al. Investigation of a novel algorithm for synchronized left ventricular pacing and ambulatory optimization of cardiac resynchronization therapy: results of the adaptive CRT trial, I807-I8I4. Copyright (2012), with permission from Elsevier. ${ }^{13}$ Abbreviations: aCRT, adaptive cardiac resynchronization therapy; CCC, concordance correlation coefficient. 
Table I Secondary end points of aCRT study

\begin{tabular}{|c|c|c|c|c|c|c|}
\hline & \multicolumn{2}{|c|}{$\operatorname{aCRT}(n=3 \mid 8)$} & \multicolumn{2}{|c|}{$\mathrm{ECHO}(n=160)$} & \multirow[t]{2}{*}{$95 \% \mathrm{Cl}$} & \multirow[t]{2}{*}{$P *$ (margin) } \\
\hline & $\mathbf{n}$ & Mean \pm SD & $\mathbf{n}$ & Mean \pm SD & & \\
\hline \multicolumn{7}{|l|}{ LVESVi $\left(\mathrm{mL} / \mathrm{m}^{2}\right)$} \\
\hline Baseline & 291 & $71.7 \pm 28.3$ & 140 & $74.0 \pm 30.9$ & & \\
\hline 6-mos post-randomization & 268 & $63.5 \pm 31.9$ & 137 & $64.7 \pm 32.7$ & & \\
\hline Paired difference at 6 mos & 250 & $\sim 8.3 \pm 23.3$ & 123 & $\sim 10.5 \pm 24.2$ & $2.3 \sim(2.8-7.4)$ & $<0.000$ I (15) \\
\hline \multicolumn{7}{|l|}{ LVEF (\%) } \\
\hline Baseline & 291 & $29.6 \pm 9.2$ & 140 & $30.3 \pm 8.4$ & & \\
\hline 6-mos post-randomization & 268 & $33.6 \pm 10.4$ & 137 & $32.9 \pm 10.1$ & & \\
\hline Paired difference at 6 mos & 250 & $3.9 \pm 10.0$ & 123 & $2.9 \pm 9.8$ & $1.0 \sim(1.2-3.1)$ & $0.0009 \sim(2.5)$ \\
\hline \multicolumn{7}{|l|}{ NYHA } \\
\hline Baseline & 318 & $3.0 \pm 0.2$ & 160 & $3.0 \pm 0.3$ & & \\
\hline 6-mos post-randomization & 296 & $2.0 \pm 0.8$ & 153 & $2.2 \pm 0.8$ & & \\
\hline Paired difference at 6 mos & 296 & $\sim 1.0 \pm 0.8$ & 153 & $\sim 0.8 \pm 0.8$ & $\sim 0.15(0.3-0.0)$ & $<0.000 \mathrm{I}(0.3)$ \\
\hline \multicolumn{7}{|l|}{ 6-min walk (meters) } \\
\hline Baseline & 312 & $276.8 \pm 127.5$ & 156 & $277.7 \pm 137.8$ & & \\
\hline 6-mos post-randomization & 288 & $325.5 \pm 130.4$ & 146 & $311.4 \pm 152.0$ & & \\
\hline Paired difference at 6 mos & 284 & $42.4 \pm 103.3$ & 142 & $29.0 \pm 123.0$ & |3.4 (8.9-35.7) & $0.0002 \sim(30)$ \\
\hline \multicolumn{7}{|l|}{ MLWHF QOL } \\
\hline Baseline & 286 & $48.5 \pm 24.1$ & 142 & $46.3 \pm 23.6$ & & \\
\hline 6-mos post-randomization & 263 & $28.2 \pm 22.0$ & 139 & $28.4 \pm 23.0$ & & \\
\hline Paired difference at 6 mos & 261 & $\sim 19.3 \pm 20.7$ & 135 & $\sim 17.6 \pm 23.8$ & $\sim 1.7 \sim(6.3-2.8)$ & $0.002(5.1)$ \\
\hline
\end{tabular}

Notes: *P-value for noninferiority between aCRT and ECHO arms. Reprinted from Heart Rhythm, 9(II), Martin DO, Lemke B, Birnie D, et al. Investigation of a novel algorithm for synchronized leftventricular pacing and ambulatory optimization of cardiac resynchronization therapy: results of the adaptive CRT trial, I807-I8I4. Copyright (2012), with permission from Elsevier. ${ }^{13}$

Abbreviations: aCRT, adaptive cardiac resynchronization therapy; Cl, confidence interval; LVEF, left ventricular ejection fraction; LVESVi, LV end-systolic volume index; min, minute; MLWHF, Minnesota Living With Heart Failure; mos, months; NYHA, New York Heart Association; QOL, quality of life; SD, standard deviation; ECHO, echocardiographic optimization.

( $\mathrm{n}=485)$ derived from the CRT arms of four clinical trials (MIRACLE, MIRACLE ICD, PROSPECT, and InSync III Marquis), which each provided $\mathrm{BiV}-0$ pacing. ${ }^{14}$ The study end point was to assess change in the CCS at 6-month follow-up. Patients in the HC underwent echocardiography-guided AV optimization. The adjusted absolute difference (in percent) improved in CCS between the aCRT and HC arms was $11.9 \%$

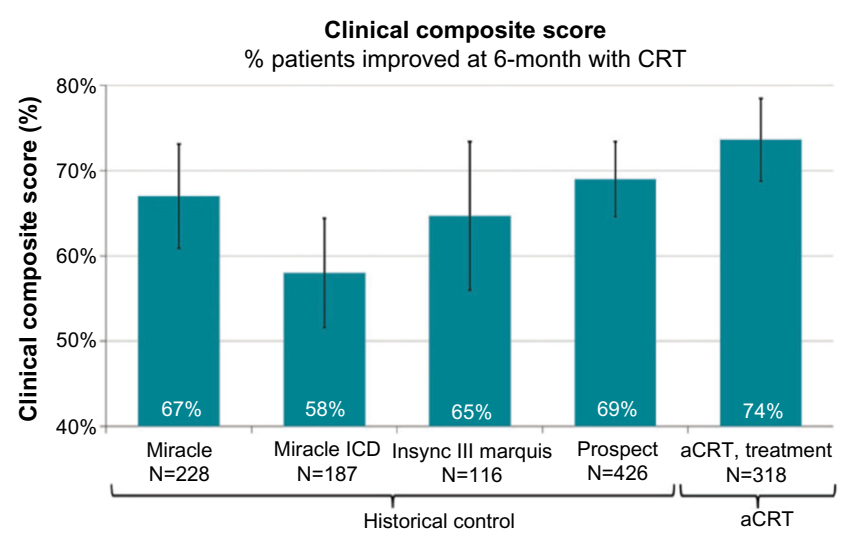

Figure 4 Percent of patients improved in CCS during 6-month follow-up.

Notes: Reprinted from Singh JP, Abraham WT, Chung ES, et al. Clinical response with adaptive CRT algorithm compared with CRT with echocardiography optimized atrioventricular delay: a retrospective analysis of multicentre trials. Europace. 2013;15(II):1622-1628 by permission of Oxford University Press. ${ }^{14}$

Abbreviation: aCRT, adaptive cardiac resynchronization therapy; ICD, implantable cardioverter-defibrillator; CCS, Clinical composite score.
(95\% confidence interval [CI]: $2.7 \%-19.2 \%$ ) favoring aCRT, and aCRT patients were significantly more likely to have an improved CCS when compared to $\mathrm{HC}$ (odds ratio $[\mathrm{OR}]=1.65$, 95\% CI: 1.1-2.5) (Figure 4). The implication from this study is that aCRT is better, rather than noninferior, to echo-guided AV optimization.

In the second post hoc analysis, patient outcomes in the aCRT trial were correlated to percent of synchronized LV

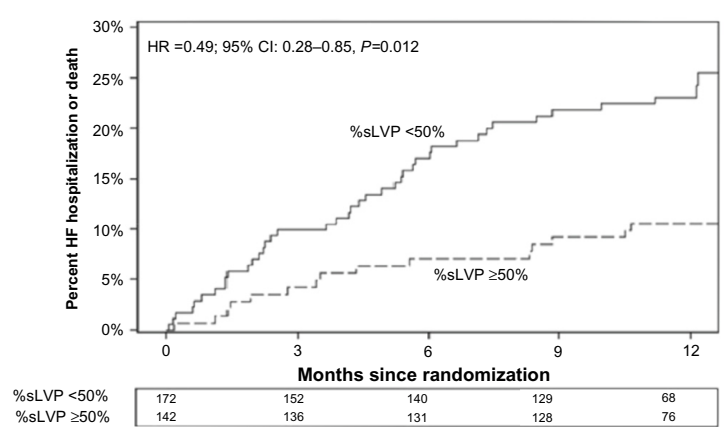

Figure 5 Time to all-cause death or first heart failure hospitalization over the I2-month follow-up stratified by percent synchronized left ventricular pacing in the aCRT arm.

Notes: Reprinted from Heart Rhythm, 10(9), Birnie D, Lemke B, Aonuma K, et al. Clinical Outcomes with synchronized left ventricular pacing: analysis of the adaptive CRT trial, 1368-1374. Copyright (2013), with permission from Elsevier. ${ }^{12}$ Abbreviations: $\mathrm{Cl}$, confidence interval; aCRT, adaptive cardiac resynchronization therapy; HF, heart failure; HR, hazard ratio; \%sLVP, synchronized left ventricular pacing as a percentage of total ventricular pacing over the I2-month follow-up. 


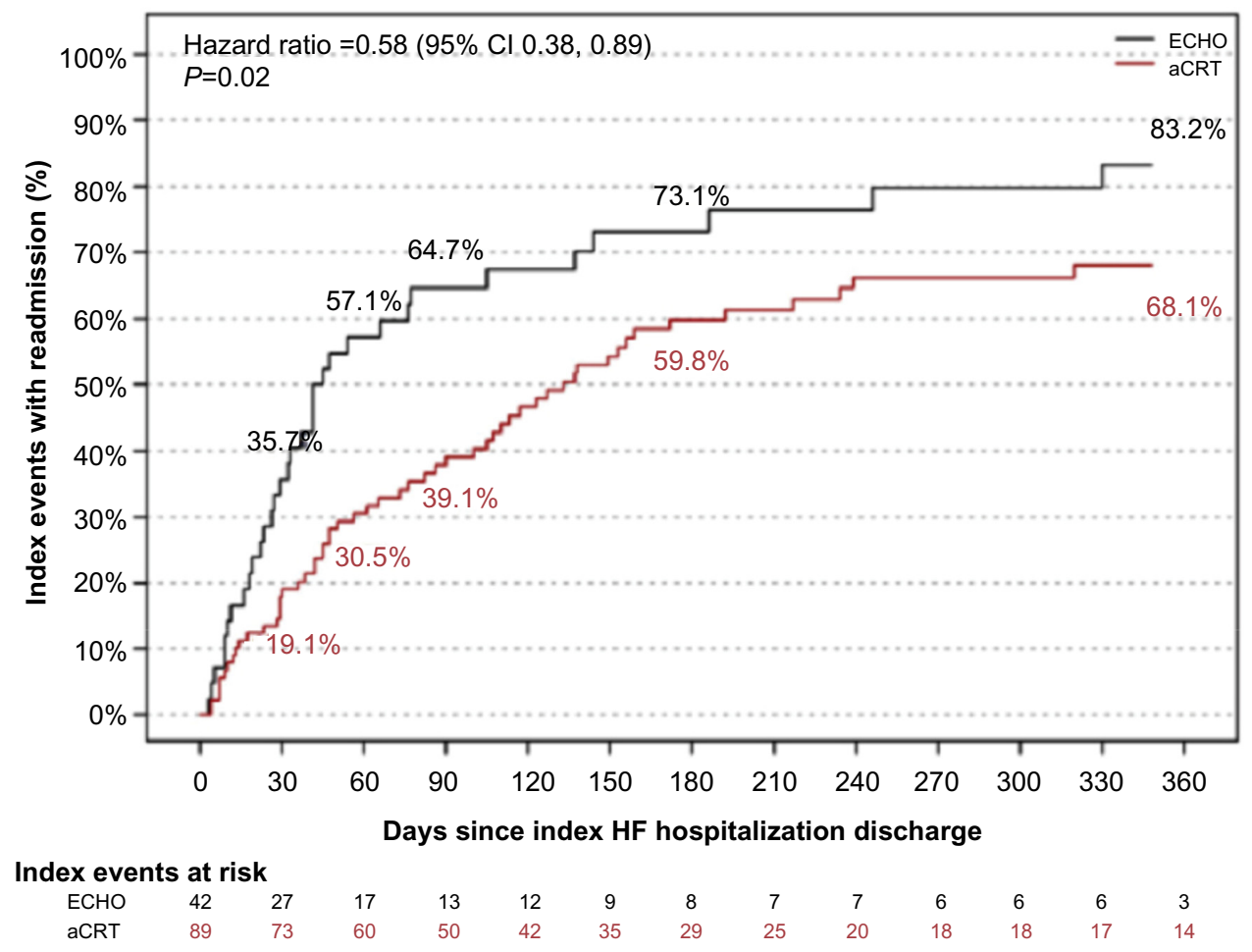

Figure 6 Time from heart failure hospitalization to all-cause readmission.

Notes: This figure was published in J Am Coll Cardiol HF, 3(7), Starling RC, Krum H, Bril S, et al. Impact of a Novel Adaptive optimization algorithm on 30-day readmissions evidence from the adaptive CRT trial, 565-572. Copyright Elsevier (20I5). ${ }^{15}$

Abbreviations: aCRT, adaptive cardiac resynchronization therapy; $\mathrm{ECHO}$, echocardiographic optimization; $\mathrm{HF}$, heart failure; $\mathrm{Cl}$, confidence interval.

pacing. ${ }^{12}$ Outcomes were also compared in the aCRT group versus control patients (BiV-0 pacing) stratified by intrinsic $\mathrm{AV}$ interval. When synchronized LV pacing was $\geq 50 \%$ in the aCRT group $(n=142 / 314,45 \%)$, there was a significant reduction in risk of death or $\mathrm{HF}$ hospitalization (hazard ratio [HR] 0.49; 95\% CI 0.28-0.85; $P=0.012$ ) compared to when synchronized LV pacing was $<50 \%$ (Figure 5). Also, in the patients with normal AV conduction ( $\mathrm{n}=241)$, the risk of $\mathrm{HF}$ hospitalization was lower in those patients treated with aCRT rather than BiV-0 (HR 0.52; 95\% CI 0.27-0.98; $P=0.044$ ). This study concluded that a greater degree of synchronized LV pacing and aCRT in patients with normal AV intervals were independently associated with improved clinical outcomes.

Starling et al completed a prospective evaluation of the original aCRT study population, assessing hospitalization for $\mathrm{HF}$ and for all causes with readmission within 30 days. ${ }^{15}$ he mean follow-up was 20.2 months, far greater than the 9.7-month follow-up in the original aCRT study. For HF hospitalizations, the 30-day readmission rate was $19.1 \%$ (17 of 89) in the aCRT group and $35.7 \%$ (15 of 42 ) in the ECHO group (BiV-0 pacing) (OR 0.41; 95\% CI 0.19-0.86; $P=0.02$ ) (Figure 6). For all-cause hospitalization, the 30-day readmission rate was $14.8 \%$ (35 of 237) in the aCRT group compared with $24.8 \%$ (39 of 157) in the ECHO group (OR 0.54; $95 \%$ CI
$0.31-0.94 ; P=0.03)$. The conclusion was that aCRT therapy was associated with a significant reduction in 30-day readmission after both HF and all-cause hospitalizations.

\section{Conclusion and clinical implications}

Modifying electrical activation of the heart with CRT has been demonstrated to be successful at enhancing cardiac performance. Yet, this field is still young and the ideal methods for electrical stimulation are unclear. An initial approach for optimization utilized echocardiography to guide electrical events by measuring changes in mechanical activation. Yet this technique has modest benefit. Another attempt to improve electrical stimulation is with electrocardiographic optimization, but this is limited by variable response and is a one-time device programming.

Adaptive CRT represents the next step in investigating best CRT pacing techniques. The algorithm was designed to provide device-based, continuous assessment and automated dynamic alteration of electrical events. The basic premise is to avoid RV pacing in patients with normal AV and right bundle conduction, and in this manner provide only the required therapy, synchronized LV stimulation, to help correct delayed left bundle activation. Simultaneous BiV pacing is utilized for patients with poor AV node - right 
bundle conduction. Adaptive CRT, therefore, is another step toward favoring LV stimulation. ${ }^{16}$ Although clinical trials to date demonstrate improved outcomes, including mortality, with aCRT, the entirety of the findings is based upon one randomized trial. Also, aCRT is only applicable in $\sim 40 \%$ of recipients of CRT, since it requires sinus rhythm, and intact $\mathrm{AV}$ node and right bundle conduction. Before embracing and building upon aCRT as the foundation for a new direction of electrical stimulation for HF, larger multicenter, randomized trials are necessary to not only confirm the benefits of aCRT, but also to compare aCRT to LV-only pacing. We also need to keep in mind the financial impact of the newer technology in CRT including adaptive CRT. The cost of the aCRT device compared to the nonadaptive CRT device is slightly higher. Our hospital purchasing department pays an extra $\$ 2,070$ for the aCRT system.

\section{Disclosure}

Mahmoud Houmsse receives researching funding from Medtronic. The authors report no other conflicts of interest in this work.

\section{References}

1. Mullens W, Grimm RA, Verga T, et al. Insight from a cardiac resynchronization optimization clinic as part of a heart failure disease management program. J Am Coll Cardiol. 2009;53(9):765-773.

2. Merchant FM, Heist EK, McCarty D, et al. Impact of segmental left ventricle lead position on cardiac resynchronization therapy outcomes. Heart Rhythm. 2010;7(5):639-644.

3. Gras D, Gupta MS, Boulogne E, Guzzo L, Abraham WT. Optimization of AV and VV delays in the real-world CRT patient population: an international survey on current clinical practice. Pacing Clin Electrophysiol. 2009;32(Suppl S1):236-239.

4. The DAVID Trial Investigators. Dual-chamber pacing or ventricular backup pacing in patients with an implantable defibrillator: the Dual Chamber and VVI Implantable Defibrillator (DAVID) trial. JAMA. 2002;288(24):3115-3123.
5. Van Gelder BM, Bracke FA, Meijer A, Pijls NH. The hemodynamic effect of intrinsic conduction during left ventricular pacing as compared to biventricular pacing. J Am Coll Cardiol. 2005;46(12):2305-2310.

6. Lee KL, Burnes JE, Mullen TJ, Hettrick DA, Tse HF, Lau CP. Avoidance of right ventricular pacing in cardiac resynchronization therapy improves right ventricular hemodynamics in heart failure patients. J Cardiovasc Electrophysiol. 2007;18(5):497-504.

7. Khaykin Y, Exner D, Birnie D, Sapp J, Aggarwal S, Sambelashvili A. Adjusting the timing of left-ventricular pacing using electrocardiogram and device electrograms. Europace. 2011;13:1464-1470.

8. Krum H, Lemke B, Birnie D, et al. A novel algorithm for individualized cardiac resynchronization therapy: rationale and design of the adaptive CRT trial. Am Heart J. 2012;163(5):747-752.

9. Rossillo A, Verma A, Saad EB, Corrado A, Gasparini G, Marrouche NF. Impact of coronary sinus lead position on biventricular pacing: mortality and echocardiographic evaluation during long-term follow-up. J Cardiovasc Electrophysiol. 2004;15(10):1120-1125.

10. Ellenboogen KA, Gold MR, Meyer TE, et al. Primary results from the SmartDelay determined AV optimization: a comparison to other AV delay methods used in cardiac resynchronization therapy (SMART-AV) trial: a randomized trial comparing empirical, echocardiography-guided, and algorithmic atrioventricular delay programming in cardiac resynchronization therapy. Circulation. 2010;122(25):2660-2668.

11. Abraham WT, León AR, Sutton MGSJ, et al. Randomized controlled trial comparing simultaneous versus optimized sequential interventricular stimulation during cardiac resynchronization therapy. Am Heart J. 2012;164(5):735-741.

12. Birnie D, Lemke B, Aonuma K, et al. Clinical Outcomes with synchronized left ventricular pacing: analysis of the adaptive CRT trial. Heart Rhythm. 2013;10(9):1368-1374.

13. Martin DO, Lemke B, Birnie D, et al. Investigation of a novel algorithm for synchronized leftventricular pacing and ambulatory optimization of cardiac resynchronization therapy: results of the adaptive CRT trial. Heart Rhythm. 2012;9(11):1807-1814.

14. Singh JP, Abraham WT, Chung ES, et al. Clinical response with adaptive CRT algorithm compared with CRT with echocardiography optimized atrioventricular delay: a retrospective analysis of multicentre trials. Europace. 2013;15(11):1622-1628.

15. Starling RC, Krum H, Bril S, et al. Impact of a Novel Adaptive optimization algorithm on 30-day readmissions evidence from the adaptive CRT trial. J Am Coll Cardiol HF. 2015;3(7):565-572.

16. Thibault B, Ducharme A, Harel F, et al. Left ventricular versus simultaneous biventricular pacing in patients with heart failure and a QRS complex >120 milliseconds. Circulation. 2011;124(25):2874-2881.
Medical Devices: Evidence and Research

\section{Publish your work in this journal}

Medical Devices: Evidence and Research is an international, peerreviewed, open access journal that focuses on the evidence, technology, research, and expert opinion supporting the use and application of medical devices in the diagnosis, treatment and management of clinical conditions and physiological processes. The identification of novel

\section{Dovepress}

devices and optimal use of existing devices which will lead to improved clinical outcomes and more effective patient management and safety is a key feature. The manuscript management system is completely online and includes a quick and fair peer-review system. Visit http://www. dovepress.com/testimonials.php to read real quotes from authors. 